\title{
Static/dynamic Analysis and Optimization of Z-axis Stand of PCB CNC Drilling Machine*
}

\author{
Yanjun Zhou ${ }^{1}$, Changtao Cai ${ }^{1}$, Songlin Zhou ${ }^{1}$ \\ ${ }^{1}$ College of Mechanical Engineering, Xihua University, ChengDu 610039, China
}

\begin{abstract}
The finite element analysis is used for the static and dynamic analysis of the $\mathrm{Z}$ axis brace of PCB CNC drilling machine. With its results of maximum displacement deformation and von Mises stress and modal frequency, the defect of original design was found out. On such bases, a variety of optimization scheme is put forward and the best size of the $\mathrm{Z}$ axis brace is obtained by the performance comparison of the schemes. This method offers bases for the design and renovation of other machine tool components.
\end{abstract}

Keywords: PCB Dynamic/static characteristics Finite element analysis Structure optimization

\section{Introduction}

Nowadays,by the rapid development of the PCB industry and electronic technology, the electronic products gradually come to the road of "multi-functional, compact, lightweight", which has forced the PCB processing (printed circuit board) to be transformed into a multi-layer, high-density, high-precision direction.PCB CNC drilling machine is specially used to process. Z-axis drilling mechanism is the most important part of impacting drilling accuracy,Its speed, positioning accuracy and the processing capacity of the tiny holes in high-speed operation are PCB CNC drilling measure important technical indicators ${ }^{[1]}$. China has played an important role in PCB manufacturing industry. Facing the various processing requirements of $\mathrm{PCB}$ from global enterprises, the native $\mathrm{PCB}$ processing equipment manufactures have to innovate technologies and upgrade the equipment to achieve products of high precision, high efficiency, high stability,high-quality.In order to achieve this, it must guarantee the quality of the processing technic for the prime segment of PCB processing, which requires not only to increase the speed of drilling spindle, feed rate, and positioning accuracy, but also to be supported by the reasonably stable mechanical structure.Therefore, how to ensure the precision of machine hole which processed by the spindle under the condition of being rotated at a high speed and high inertia, is currently a crucial technical problem in the PCB drilling industry ${ }^{[2]}$.
In the process of $\mathrm{PCB}$ board processing, the drilling plays the most important role. So, $\mathrm{Z}$ axis of drilling is the most critical part of $\mathrm{PCB}$ CNC drilling machine. Nevertheless, the Z-axis stand is an important part of Zaxis, and its rigidity and dynamic stability directly affect the accuracy of the spindle machining. With the help of the finite element analysis software, this article is intended to make a static and dynamic analysis of a certain type of PCB CNC drilling machine, on the basis of which the structure is improved for a better static and dynamic characteristics of the improved components.

\section{Model analysis}

\section{1finite element model}

The $\mathrm{Z}$ axis of PCB CNC drilling machine is mainly composed of drive motor, the spindle, $Z$ axis stand and so on. The $\mathrm{Z}$ axis assembly is shown in Figure 1.The $\mathrm{Z}$ axis stand is connected to the slider on the beam guide rail by bolts and by which the spindle and its affiliated parts are fixed in the $\mathrm{Z}$ axis stand to get the movements in $\mathrm{X}$ direction.So,the $\mathrm{Z}$-axis stand is key part of $\mathrm{Z}$-axis.

* Sichuan province science and technology support project (15ZC2176) 


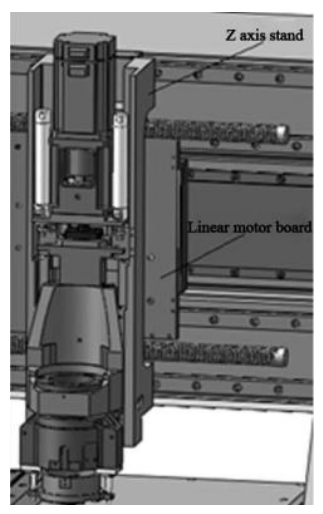

Fig.1 $\mathrm{Z}$ axis assembly

Take advantage of the Solid-works 3D software to model the $\mathrm{Z}$ axis stand. The $\mathrm{Z}$ axis stand is casted with the material 6061 alloy. To ensure the accuracy and improve the efficiency of the analysis, the model can be appropriately simplified without affecting the whole analysis, including removing the small chamfer, fillet and threaded hole which has no effections on parts' rigidities. When chose the solid mesh for the parts to make the gridding division, increasing the number of units can improve the accuracy. And using different meshing precision will have a different result.Then with the overall size and tolerance are $10 \mathrm{~mm}$ and $0.5 \mathrm{~mm}$ standard meshing the part.There are 33,567 solid elements. The finite element model and meshing is shown in Figure 2.

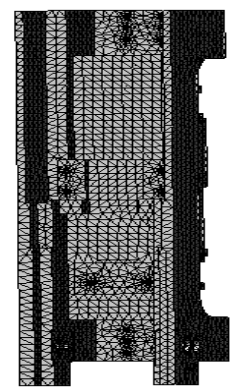

Fig.2 The finite element model

\subsection{Static analysis}

Static analysis is the structural response which the parts unit changes over the time with the action of equivalent static and changeless load. It can be used to checkout whether the rigidity and intensity of the parts meet the demands of design ${ }^{[3]}$.

The spindle and its affiliated parts are fixed on the supporting frame by bolts. This article will mainly study the static and dynamic characteristics of $\mathrm{Z}$ axis brace in the process of marching forward $\mathrm{X}$. As the size and direction of force which produced during the principle axis motion are very complicated, the factors of main shaft and auxiliary parts will not be taken into consideration. The power by which the $\mathrm{Z}$ axis brace marches in $\mathrm{X}$ directions is directly offered by the liner motor, making it to the expected position quickly, smoothly and accurately. So, by the calculation, the maximum thrust which the brace bears is: $F x=2500 N$. the gravity of stand is far less than the thrust because of material properties and the particularity of parts. So,the article didn't taken the gravity of stand into account. The $\mathrm{Z}$ axis stand is connected to the slider on the beam guide rail by bolts,constraining the screw hole which corresponds to the slider and the stand to achieve the constraint of the stand.Completion of the $\mathrm{Z}$ axis stand load is applied after all its constraints static analysis.

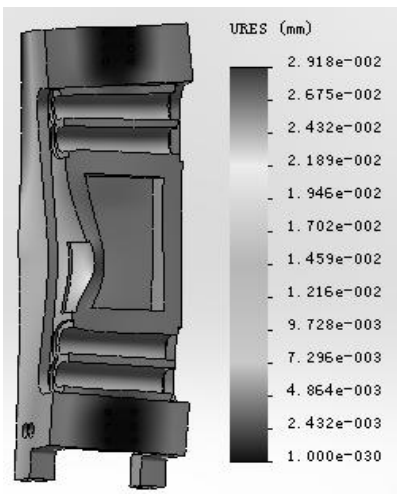

Fig.3 static displacement

The static displacement is shown figure 3.The maximum deformation of $\mathrm{Z}$ axis stand is located in the thin-wall of right side and of which the amount of it is $0.0292 \mathrm{~mm}$. However, PCB CNC drilling machines allowed error range is within $0.02 \mathrm{~mm}$. So it is not enough to meet the static stiffness requirements and should be improved the structure size to reduce the amount of deformation in the optimization process.The first main stress of the stand is shown in Figure 4.The maximum stress did not exceed the intensity value even though it appeared in the same place with the maximum deformation.From the view of stress analysis, the ability of the material to resist external damage is good.

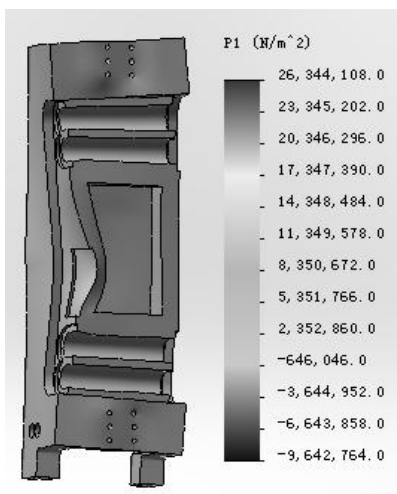

Fig 4 The first main stress

\subsection{Dynamic analysis}

The modal analysis is a method of understanding vibration characteristics of the structure which is one of the indispensable step of dynamic analysis.The characteristic parameters of mechanical structure can be obtained by modal analysis that can analyze the structure 
of defects then taking measures to avoid resonance or fatigue to structural.

The modal analysis carried on Z-axis stand using the same finite element model of statics. This is the dynamic characteristics analysis of Z-axis stand which is to know the natural frequency and modal shape of stand regardless of its loading conditions. The machine tool structural damping has little effect on the dynamic performance of the machine.So,the machine can be regarded as a $n$ degrees of freedom of without oscillation system which can be expressed as follows,

$$
[M]\{x\}+[K]\{x\}=\{F\}
$$

Among them, $[\mathrm{M}]$ is a mass matrix of vibration system and $[\mathrm{K}]$ is a stiffness matrix; $\{\mathrm{F}\}$ is a outside stimulation matrix and it is 0 matrix in this formula. $\{x\}$ 和 $\{x\}$ are acceleration and displacement matrix of the points. By solving this equation can be drawn the following relationship,

$$
f_{i}=(1 / 2 \pi) \sqrt{k_{i} / m_{i}}
$$

In formula (2), the natural frequency $f_{i}$ is in direction proportion to $\sqrt{k_{i} / m_{i}}$, which showed the greater $f_{i}$ is, the higher stiffness of unit mass is.So, improving the natural frequency means increasing stiffness of the brace while its dynamic performance also will improve. Now using the finite element software analyzed on the stand and by changing the shape or size of the model to increase its natural frequency and dynamic performance.

In engineering applications, the only thing needed is to focus on the first few natural frequencies.Because the precision problem of large matrix eigenvalue calculation and the sensitive changes of the higher-order frequency with simplified model, makes the natural frequency of the high order don't have much reference value[4].Therefore, by using the finite element analysis software obtained the first four natural frequencies and mode shapes. The result are shown Figure 5 and Table 1.

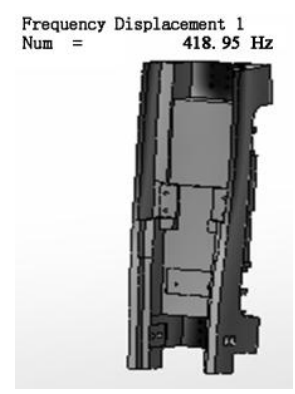

(a) $1^{\text {th }}$ mode shape

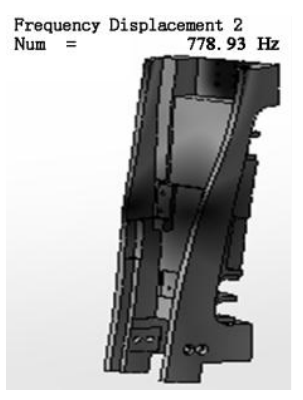

(b) $2^{\text {th }}$ mode shape

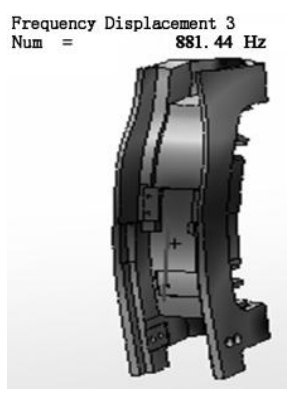

(c)3h mode shape

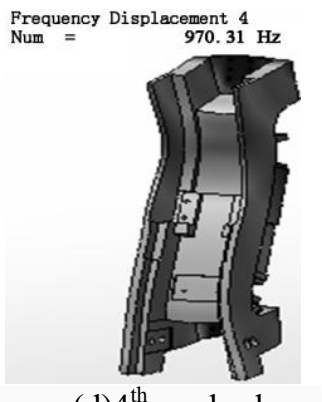

(d) $4^{\text {th }}$ mode shape

Fig.5 Mode shape of the stand

Table 1 First four natural frequencies and mode shapes

\begin{tabular}{ccc}
\hline stage & freuency & Mode shapes \\
\hline $1^{\text {th }}$ & $419 \mathrm{~Hz}$ & swing \\
\hline $2^{\text {th }}$ & $779 \mathrm{~Hz}$ & Twist around \\
\hline $3^{\text {th }}$ & $881 \mathrm{~Hz}$ & Back and forth \\
\hline $4^{\text {th }}$ & $970 \mathrm{~Hz}$ & Bobbing \\
\hline
\end{tabular}

As can be seen from table 1 , the $\mathrm{Z}$-axis stand can completely avoid the resonance because the first natural frequency of $419 \mathrm{~Hz}$ is far than the excitation frequency of the spindle.The inherent frequencies of the later three steps changed slightly, and then basically leveled off. So,the dynamic characteristics of the stand is good by the first four order natural frequencies.

\section{Optimization of Z-axis stand}

\subsection{Size optimization of stand}

From the above results, the Z-axis stand had better dynamic performance and poor static performance.The 
structural optimization aimed at increasing the performance of static characteristics under the conditions of without lowering the performance of the dynamic characteristics. Learn from the working state of the stand, the main load in its right behind thin wall where is the biggest place of static deformation. In order to increase the static stiffness of stand in working condition can be properly thickened the right behind thin wall. (1)Improved type a,the right and left thin wall was thickened to about $31 \mathrm{~mm}$ then the upper and lower unchanged;(2)Improved type $b$,the right and left thin wall was thickened to about $31 \mathrm{~mm}$ then the upper and lower was reduced to $14 \mathrm{~mm}$; (3)Improved type $\mathrm{c}$, the right and left thin wall was thickened to about $41 \mathrm{~mm}$ then the upper and lower unchanged;(4)Improved type $d$, the back groove of stand was deleted.

\subsection{Performance Comparison}

The improved types were analyzed under the same conditions as before. The results are shown in Table 2 .

As can be seen from the table,comparing with the original model, the change of the natural frequency is not obvious and it does not affect the dynamic rigidity. Theirs static rigidity had certain improvement.Among the static and dynamic characteristics analysis, four improved schemes are feasible. But the improved type $a$ and type $b$ that did not make the center of gravity above the safe range. And the improved typed greatly increase the weight. Therefore, considering the problem of the weight and the center of gravity, the improved type c should be used.

Table 2 static/Dynamic analysis and comparison

\begin{tabular}{lccccccc}
\hline & \multicolumn{3}{c}{ Natural frequencies/Hz } & $\begin{array}{c}\text { Compreh } \\
\text { ensive } \\
\text { displace } \\
\text { ment } / \mathrm{mm}\end{array}$ & $\begin{array}{l}\text { weight/ } \\
\mathrm{kg}\end{array}$ & $\begin{array}{l}\text { Gravity } \\
\text { positio } \\
\mathrm{n} / \mathrm{mm}\end{array}$ \\
\hline & $1^{\text {th }}$ & $2^{\text {th }}$ & $3^{\text {th }}$ & $4^{\text {th }}$ & & & \\
\hline $\begin{array}{l}\text { The } \\
\text { original }\end{array}$ & 419 & 779 & 818 & 970 & 0.0292 & 9.203 & 13.607 \\
\hline type a & 418 & 777 & 877 & 955 & 0.0169 & 9.545 & 14.418 \\
\hline type b & 418 & 778 & 879 & 963 & 0.0186 & 9.328 & 13.916 \\
\hline type c & 417 & 774 & 870 & 957 & 0.0139 & 9.981 & 15.172 \\
\hline type d & 416 & 771 & 866 & 949 & 0.0114 & 11.789 & 17.406 \\
\hline
\end{tabular}

\section{Conclusion}

Achieving optimization of the overall structure is very difficult for the type of sophisticated machine tools such as PCB CNC drilling machine.So, achieve overall optimization step by step through optimization of the main components of the machine.In this article, the largest deformation displacement and the first four natural frequencies and mode shapes of stand were obtained by using the finite element analysis software.According to the results, the deformation characteristics and the deficiency of the original design were found.Under the conditions of considering the problem of the weight and the center of gravity,the optimization increased the static rigidity of stand.The stand was optimized in the aspect of size and the optimized structure was compared for performance.Based on the static/dynamic analysis of the optimized structure,its overall performance has significantly improved comparing with the original structure which achieved the expected goals. It laid the foundation for optimization design of similar parts. At the same time,other parts of the machine have a greater significance.

\section{References}

1. Yingzhang Wang, Zongjun $\mathrm{Xu}$. The Development of Domestic PCB CNC Drilling Machine[J]. Machinery \& Electronics. 2004(6):66-68.

2. Bing Wang, Chengyong Wang, Hongqun Tang. The Characteristics and Key Technologies of PCB CNC Drilling Machine[J].Tool Engineering.2009:3-10.

3. Lianming Xia. The Finite Element Analysis of the Excavator on Solid-works and ANSYS[J].Mining Machinery.2012(4):32-34.

4. Luyan Qi, Yulin wang,Hutian Feng. Dynamic and Static Analysis and Atructural Optimization of Accurate Grinder Bed[J].Modular Machine Tool \& Automatic Manufacturing Technique.2012(6):9-12.

5. Yuping Yang, Sen Zhang,Binbin Ji,Zixue Qiu.Design and Analysis of the Bed Stiffened Plate Structure for Vertical Machining Center[J].Machinery Design \& Manufacture. 2013(12): 38-40.

6. Jun Qiu, Xiaohui Zhu, Wei Huang, Xiaozhou Li.Static and Dynamic Performance Analysis and Structure Improvement of Pillar in Upright Drill Press[J]. Machine Tool \& Hydraulics. 2012(7):152-155.

7. Chunlei Xu, Qingchun Zheng, Changqing Yang, Yahui Hu.Optimization Design Method Research of Vertical Precision Grinder Bed Structure Driven by Multi-objective[J]. Modular Machine Tool \& Automatic Manufacturing Technique.2013(1): 23-30. 
8. Yanhua Peng, Yisheng Guan,Xianmin Zhang,etc. Multi-objective Optimaization Design for a Vertical Machining Center Based on Composite Optimization Method[J]. Journal of Vibration and Shock. 2012(6): 48-52.
9. Yan Chen, Song Zhang, Zhou Chen,etc. Dynamic/static Characteristics Analysis and Optimization of Slide of Gantry Machining Center[J]. Design and Research. 2014(8):64-68. 